\title{
Dopamine double rules
}

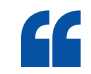

however, D1Rs

and D2Rs

modulated

rule-selectivity

in distinct ways

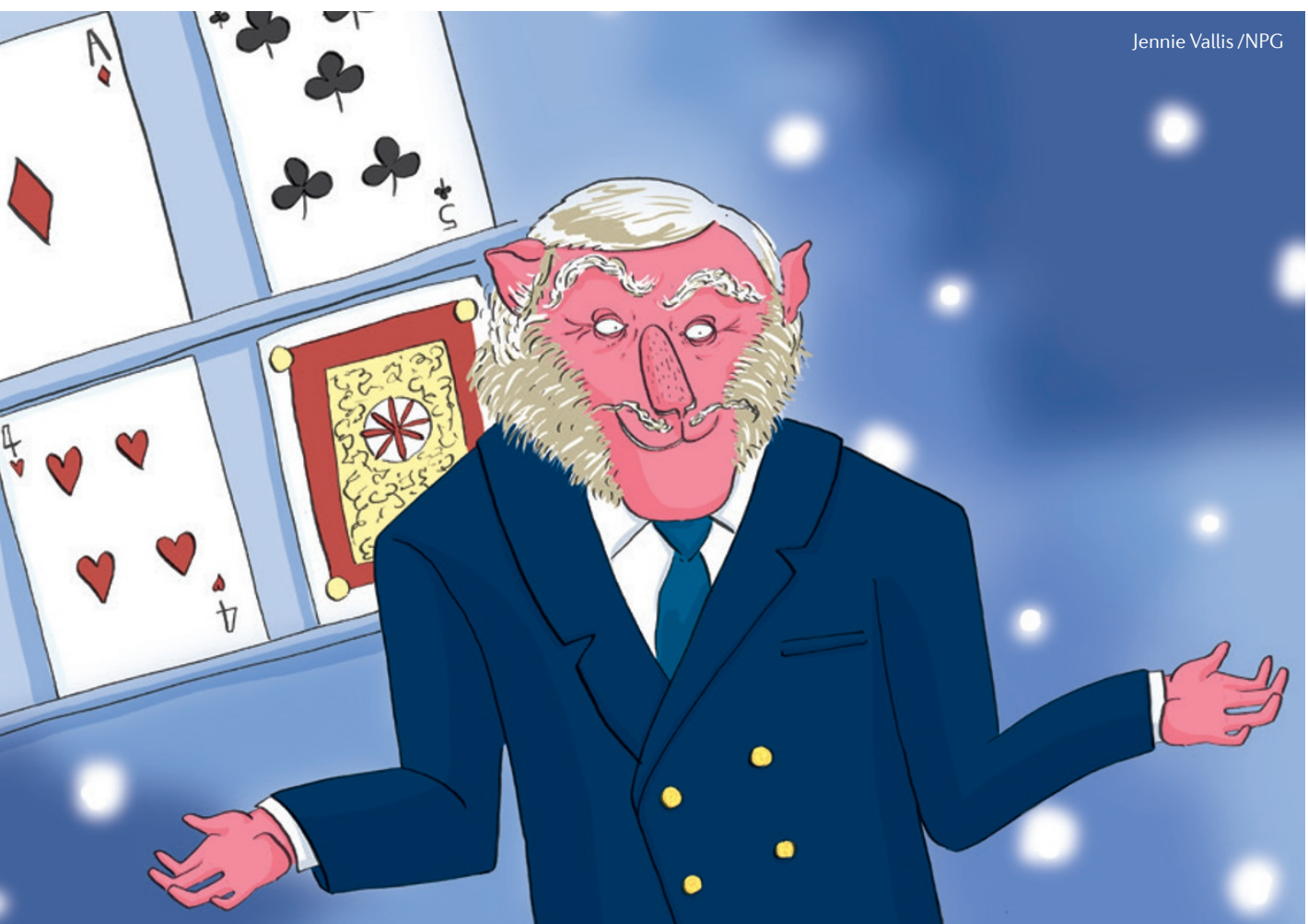

arrangement of dots, and then a cue (for example, a red or blue circle) that indicated which rule the monkey was to apply upon seeing a subsequently presented 'test' image, also comprising an arrangement of dots. The cues represented either a 'greater than' rule - which required the monkey to respond by releasing a lever if there were more dots in the test than in the sample image - or a 'less than' rule, which required the same response, but only if the test image contained fewer dots than the sample image. This task tests abstract-rule encoding, as well as executive flexibility (to switch between rule-based motor plans).

During the task, the authors recorded the activity from $384 \mathrm{PFC}$ neurons across the two monkeys. The D1R agonist SKF81297, the sented Specifically the monkeys were shown a 'sample' image, comprising an

The authors trained two monkeys on a numerical rule-switching task, in which the animals had to apply different rules depending on the cues pre-
D1R antagonist SCH23390 or the D2R agonist quinpirole was iontophoretically applied to the recording site during the task to investigate the separate contributions of PFC D1Rs and D2Rs to executive functioning.

The authors identified PFC neurons that showed 'rule-selectivity' - that is, neurons that exhibited higher spike rates in response to one of the rules, but not for any particular quantity of dots in the sample images or rule-cue modality. Thirty PFC neurons were selective for the 'greater than' rule, and thirty-four were selective for the 'less than' rule. When applied to the PFC, the D1R agonist and the $\mathrm{D} 2 \mathrm{R}$ agonist increased the ruleselectivity of these neurons: the difference between the spike rates elicited by the preferred rule versus the non-preferred rule was wider. By contrast, the D1R antagonist diminished rule selectivity in these neurons. Intriguingly, however, D1Rs and D2Rs modulated ruleselectivity in distinct ways. Whereas D1R-specific stimulation led to enhanced neuronal activity in response to the preferred rule (without modulating responses to the non-preferred rule), D2R-specific stimulation suppressed neuronal responses to the non-preferred rule (but did not affect responses to the preferred rule).

Together, these results show that D1Rs and D2Rs in the PFC contribute to rule-based executive function, and that they do so through different but complementary effects.

Natasha Bray

\section{ORIGINAL RESEARCH PAPER Ott, T. et al.} Dopamine receptors differentially enhance rule coding in primate prefrontal cortex neurons. Neuron http://dx.doi.org/10.1016/j. neuron.2014.11.012 (2014) 